\title{
Acute endodontic and dental trauma provision during the COVID-19 crisis
}

\author{
Khawer Ayub*1 and Aws Alani ${ }^{1}$
}

\section{Key points}

Provides an insight into the impact of COVID-19 on dentistry and the potential risks of aerosol generating procedures.
Discusses common endodontic emergencies likely to present during COVID-19.
Suggests a management protocol for endodontic emergencies and a brief management protocol for dento-alveolar trauma.

\begin{abstract}
COVID-19 (also known as coronavirus) has had a profound impact on dentistry, with all elective treatment grinding to a halt initially and a slow phased return to normal services. The guidance available regarding treating dental emergencies is currently advice, analgesia and antimicrobials, where indicated in the first instance. Some patients cannot be managed successfully with this approach and require face-to-face consultation. Unless dental practices have the appropriate PPE and follow strict infection prevention and control guidance, face-to-face consultation may not be possible. If treatment is required, this is based on minimum intervention and avoidance of aerosol generating procedures (AGPs) on patients who are free of COVID-19. COVID-19-positive or suspected patients still require treatment at designated Urgent Dental Care centres.

The aim of this article is to provide an insight into the COVID-19 pandemic and its implications on current emergency dental care. Commonly presenting dental conditions which require endodontic management will be discussed. Finally, an endodontic management protocol is suggested. The intention of the protocol is to describe practical techniques to minimise potential viral load and reduce risk of COVID-19 transmission when AGPs are instigated.
\end{abstract}

\section{Introduction}

In late December 2019, an evolving disease was spreading from Wuhan City, China, known as coronavirus disease 19 (COVID19). It was initially rumoured to be a lowgrade, common flu-like virus; however, it is now understood to be at least twice as infective as the common flu. ${ }^{1}$ COVID-19 is caused by a single-strand RNA SARS-CoV-2; its genome sequence closely resembles that of the severe acute respiratory syndrome coronavirus (SARS-CoV) and the Middle East respiratory syndrome coronavirus (MERS$\mathrm{CoV})$. Currently, worldwide, there are over 7.2 million confirmed cases and over 411,000 deaths. ${ }^{2}$ The impact of this disease is farreaching, with government implementation

'Department of Restorative Dentistry, King's College Hospital Dental Institute, Denmark Hill, London, SE5 9RW, UK.

*Correspondence to: Khawer Ayub

Email address: khawer.ayub@nhs.net

Refereed Paper.

Accepted 18 June 2020

https://doi.org/10.1038/s41415-020-1920-0 of social distancing and, in some parts of the world, a total lockdown is in place.

COVID-19 is not heavily contagious; however, it is thought the rapid spread is via contact with infected surfaces or droplet contamination. ${ }^{3}$ Subsequently, the virus is introduced into the body via mucosal surfaces such as the nose, mouth and eyes. ${ }^{4}$ A proportion of the population will present asymptomatically or with mild symptoms. At the current time, the only realistic methods of containing the spread are to minimise close contact via social distancing, effective and frequent hand hygiene, reducing hand contact with the face, and, where necessary, appropriate surface disinfection. Reinforcing this message has been attempted by the UK government on the whole; however, unfortunately, there has still been over 40,000 deaths. ${ }^{5}$

The World Health Organisation provides a list of medical procedures which generate aerosols although this list does not include any dental aerosol generating procedures (AGPs). ${ }^{6}$ However, it has been reported in the literature that routine dentistry involves AGPs. ${ }^{7}$ Clinical dentistry involves close proximity to patients, theoretically resulting in dental professionals being more susceptible to COVID-19 infection and possible spread of the disease, although this is yet to be substantiated or extensively researched. The Office for National Statistics has produced estimations for exposure to generic diseases for UK occupations based on physical proximity to others. Dental practitioners and nurses come into close proximity with patients on a daily basis and it could be argued that they face an increased exposure to general disease and COVID-19. ${ }^{8}$ The use of personal protective equipment (PPE) and strict infection control regimes should of course negate this. Public health bodies in England and Scotland have stated that dental procedures with the use of high-speed drills and ultrasonic scalers are potentially infectious AGPs for COVID-19.9,10 Guidance has been published by these bodies for dental practitioners across these respective regions. These aerosols could potentially mix with both blood and saliva, therefore allowing airborne transmission of droplet-spread diseases like COVID-19. With the vast aerosols generated in dental surgeries and the possibility of the virus 


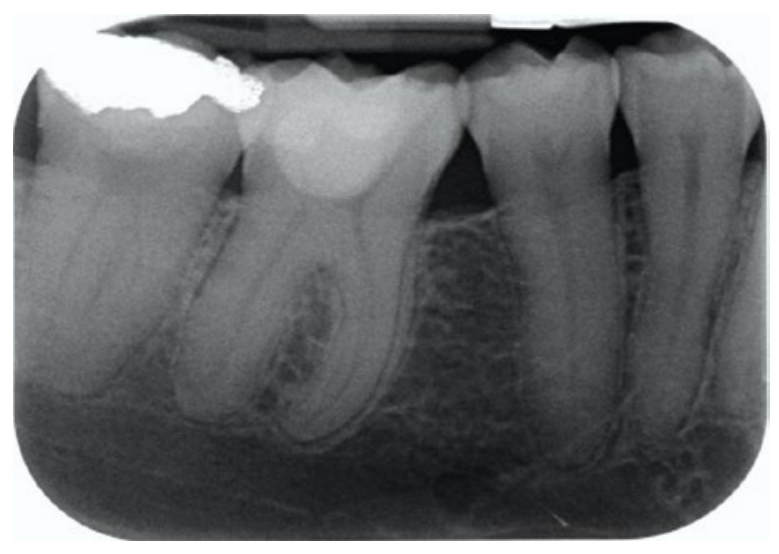

Fig. 147 (46 missing) diagnosed with irreversible pulpitis. Clinically, this tooth had a large occluso-buccal restoration; radiographically, the restoration is encroaching on the pulp

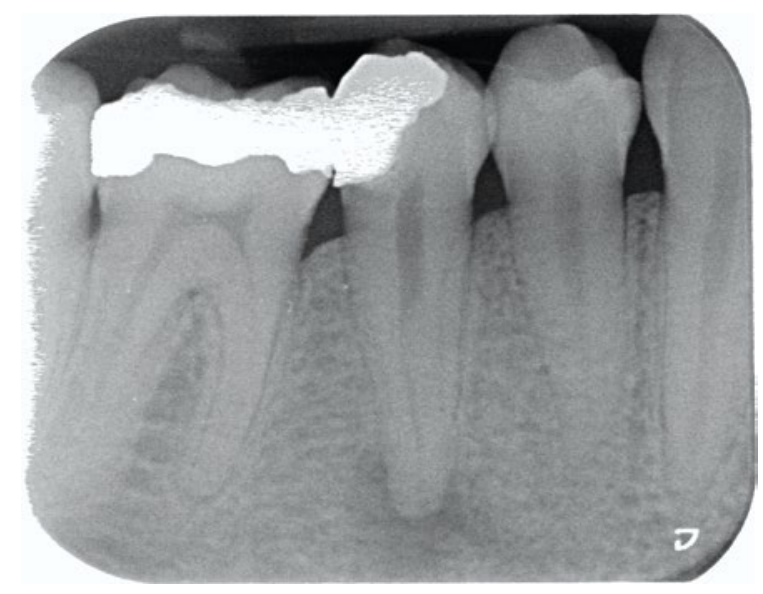

Fig. 245 diagnosed with symptomatic apical periodontitis. The periapical radiograph confirms a large periapical radiolucency

remaining active for up to three days, ${ }^{11}$ there is an increase of potential transmission from infected individuals if correct protocols are not followed when attending dental surgeries.

\section{Primary care dentistry}

On 25 March 2020, the Chief Dental Officer (CDO) advised that the provision of all nonurgent dental treatment was to be either deferred or stopped entirely. ${ }^{12}$ On the basis of no further treatment, patients would be dealt with remotely via telephone triage and treated for acute problems where possible on the basis of advice, analgesia and antimicrobials (AAA), if possible in the first instance. If the clinician, through telephone triaging, considers the condition to warrant a face-toface consultation and further treatment, then the patient would need to be referred to urgent dental care centres (UDCCs). Any treatment would be provided with the aim of reducing or avoiding AGPs unless absolutely necessary. ${ }^{13}$
An outline of conditions which are likely to require treatment include: ${ }^{14}$

- Life-threatening emergencies; for example, orofacial swellings causing airway restriction or breathing/swallowing difficulties

- Dento-alveolar trauma including facial/oral laceration and/or dento-alveolar injuries including fractures and luxations

- Severe dental and facial pain - pain that cannot be controlled by the patient following self-help advice

- Post-extraction bleeding that the patient is not able to control with local measures.

Further guidance released in May from the CDO recommended that dental practices could reopen as of 8 June $2020 .^{15}$ This was a phased transition to a return to normal service and a number of key principles were outlined. Initially, remote consultations should be provided and AAA should continue to be adopted in the first instance.
Patients who are displaying no symptoms of COVID-19 could be offered face-to-face appointments, and a range of treatment could be offered on the proviso that appropriate PPE was available and in line with infection prevention and control (IPC) guidance. If treatment is planned, then AGPs should be avoided if possible and intervention kept to a minimum. If patients are confirmed or suspected of COVID-19, they should still be referred to UDCCs. ${ }^{16}$

\section{Acute dental presentation and management}

If patients are in pain, the recommendations mentioned above should be adopted. The AAA principle previously discussed should be provided in the first instance where appropriate. This may be successful for a proportion of the cases in the short term that are managed via phone triage. In areas where systems and services are available, dental practices can instigate face-to-face consultations and subsequent treatment if IPC guidance and appropriate PPE are available; however, this may not be the case for a number of dental practices.

The commonly presenting conditions are briefly discussed below: ${ }^{17,18}$

- Irreversible pulpitis (Fig. 1) - longlasting, throbbing pain with spontaneous onset. Exacerbated by hot and relieved by cold, and is commonly poorly localised. Patients with irreversible pulpitis would have previously been given appropriate analgesic advice. Usually those attending for treatment would have been unable to manage the severe pain with analgesics alone. On occasion antimicrobials may have been prescribed which, of course, will not be effective for pulpitis. Reversible pulpitis should be able to be managed with analgesia and appropriate advice at triage

- Symptomatic apical periodontitis (Fig. 2) - dull, throbbing constant pain and is exacerbated when biting on the affected tooth. The patient is able to localise to a single tooth. These patients are often in severe pain and, unfortunately, analgesia may have been ineffective. Commonly, these patients may have been prescribed one or more antimicrobials which have previously resolved the pain

- Acute apical abscess - severe, spontaneous pain with a swelling and usually pus discharge. The tooth is well localised as it 
can be tender to bite on. If these patients present with severe symptoms, soft tissue swellings and spreading infection, then appropriate hospital referral may be required for management by the oral and maxillofacial surgery team.

\section{General considerations for management of endodontic emergencies}

Due to the need for social distancing measures and minimising the risk of transmission, specific considerations for the flow and management of patients is required. For example, a waiting room full of patients increases risk, as do areas where patients with and without COVID-19 symptoms could come into contact. In this respect, booked appointments are needed with adequate staggering of clinic times. Waiting rooms can be zoned with appropriate spacing between seats. Where waiting rooms are small, the clinician may decide to have only one patient waiting at any one time to further minimise risk. Depending on symptoms, patients who are either confirmed or potential COVID-19positive should ideally wait and be treated in different areas to those that are asymptomatic. Further consideration should be given to patients that are in vulnerable groups (those who are 70 or older, pregnant, obese, diabetic, suffer with lung or heart disease, and patients taking medications which affect the immune system), who should also be managed in different areas if possible.

The dentist and nurse should not leave the surgery during an AGP procedure due to droplet contamination, and therefore all the necessary required equipment should be laid out as best as possible to avoid any unnecessary sourcing of items. A spare 'runner' nurse should remain outside the surgery to provide any further materials required intra-operatively. These materials can be transferred via a trolley from outside the surgery.

Prior to any AGPs, appropriate PPE should be put on (donning) in a separate area/surgery. Practically, nurse and operator can aid one another when donning PPE to ensure it is correctly placed, or the nurse 'runner' can be used as a 'buddy'. PPE should be removed in the reverse order (doffing) and ideally in a separate dedicated area if available. If no dedicated area is available, then doffing can be completed in the patient treatment room,
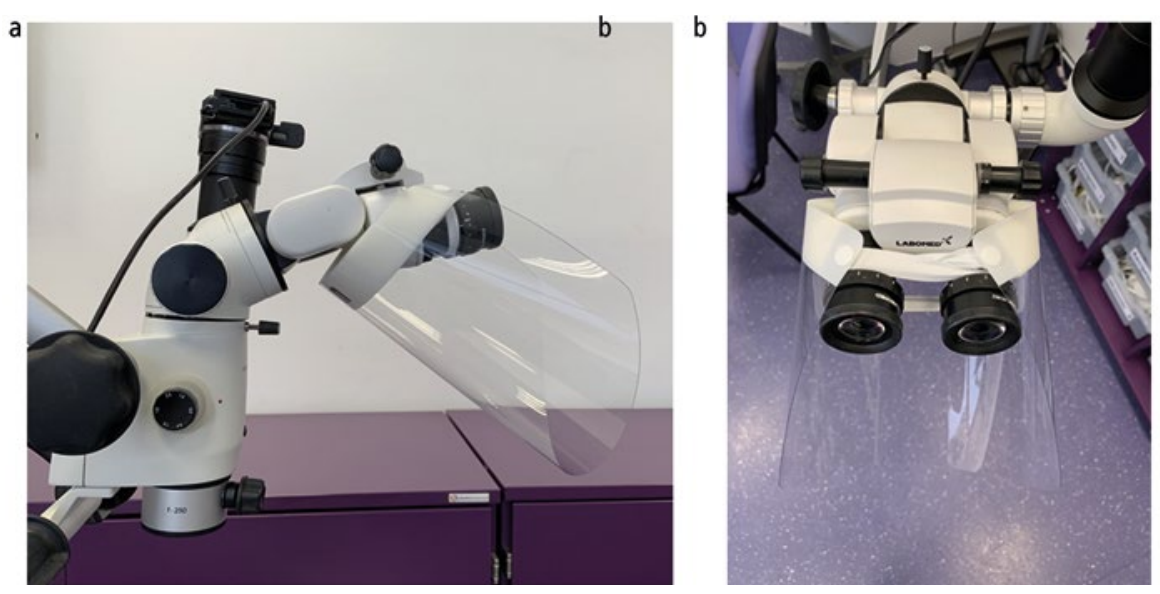

Fig. 3 Full-face visor attached to dental microscope. a) Side view. b) Front view

except for respirator masks which should be removed outside the room. Further guidance on donning and doffing is available from Public Health England. ${ }^{19}$

Following completion of any AGPs, the aerosol cloud will need to be cleared before any further use of the room. Aerosols can commonly remain in dental surgeries for between 10-30 minutes before dissipating. ${ }^{20}$ Current recommendations state surgeries should be left vacant for 20 minutes in a negative pressure room and for one hour in neutral pressure rooms. ${ }^{21}$ Following dispersion and settling of the aerosol, a meticulous clean should be completed before any further patient treatment in the same surgery. For this reason it is recommended that a number of surgeries should be available for use during AGPs. Clinicians and nurses can utilise free surgeries while waiting for terminal cleans of previously used surgeries. This should increase efficiency and could reduce numbers of patients gathering in waiting rooms.

More commonly, clinicians are utilising magnification when providing endodontic procedures; these are usually in the form of dental loupes or a microscope. This may pose a problem when using enhanced PPE and, in particular, a full-face visor. If the visor does not sit vertically, some have advocated creating holes within the visor to accommodate the loupes, but this unfortunately creates an avenue for aerosol to travel through. If dental microscopes are to be used, then appropriate barrier wrap and protection may be more of a challenge. Recommended full-face visors can be inverted and tied to the eyepiece (Fig. 3). The operator's chin can rest on the visor to provide some protection; while positioning their eyes close to the eyepiece of the microscope, a form of eye protection should be worn. Both these techniques are largely based on initiative as they do not provide the same protection as full-face visors.

More recently, new COVID-19 guidance has been issued by the Faculty of General Dental Practice (UK) and the College of General Dentistry. ${ }^{22}$ This guidance sets out the safe management of dentistry within general practice. It adopts a risk-based 'aerosol generated exposure' approach and stresses the importance of personalised risk assessments for both patients and staff. This guidance covers aspects discussed above and details the patient journey from pre-appointment to post-treatment. These factors are considered alongside best available clinical evidence and the national alert level to underpin safety for staff and patients.

Some of these views have been echoed in the standard operating procedure issued by the CDO on 4 June $2020 .{ }^{16}$ This outlines a phased approach to resumption of dental care and prioritises patients based on a risk assessment. It does stress in the first instance to continue with remote management and to minimise face-to-face contact. The document also highlights clinical guidance for a range of specialities including endodontics.

\section{Suggestions for acute endodontic management during the COVID-19 crisis}

These are suggestions for the management of patients in pain and in an emergency requiring endodontic intervention. As aseptic a technique and as little aerosol production as possible should be the aim. A further aim is optimal and efficient endodontic access 
and instrumentation. This would be in the hope that any further interventions or acute symptoms are prevented or nullified, especially during the period where services are not up to normal operational capacity.

What is vitally important is that the diagnosis is clear, the tooth is restorable in a predictable manner and the need for endodontic management is appropriate. The patient also needs to be aware that the tooth will require the completion of the endodontic treatment. If a tooth is accessed and later deemed unrestorable or the patient has the tooth extracted, then an AGP could well have been initiated unnecessarily.

\section{Pre-operative}

- Consider 'scrubbing' the patient's lips and surrounding area with povidone-iodine (PVP-I) or chlorhexidine if available to maintain as aseptic a technique as possible, similar to other dental procedures which require an aseptic technique ${ }^{23}$

- A form of mouthwash can be considered pre-operatively - gargling with PVP-I or hydrogen peroxide has the potential to reduce the salivary viral load $\mathrm{d}^{1,24,25}$

- Practically, hydrogen peroxide mouthwash may be more readily available in concentrations ranging from 1.5-9\%. This can be rinsed around the mouth for one minute

- It is thought that chlorhexidine mouthwash may not be effective against COVID-1926,27

- There is also the potential for the use of PVP-I as a nasal spray and mouthwash for both patients and healthcare workers. A $10 \%$ PVP-I solution is diluted down to $0.5 \%$ concentration. This solution is gargled in the mouth and held at the back of the throat for one minute and administered into the nostril using an atomising device or syringe. For healthcare workers, this can be repeated every two to three hours. A note of caution: PVP-I is for oromucosal use, but in this instance, it may be considered an 'offlabel' application..$^{28}$

\section{Intra-operative}

- Single use instruments to be used wherever possible to reduce the need for sterilisation and decontamination

- Reduce the need to utilise intraoral radiographs wherever possible - this is unlikely to be practical for endodontics, as an appropriate pre-operative radiograph should be available
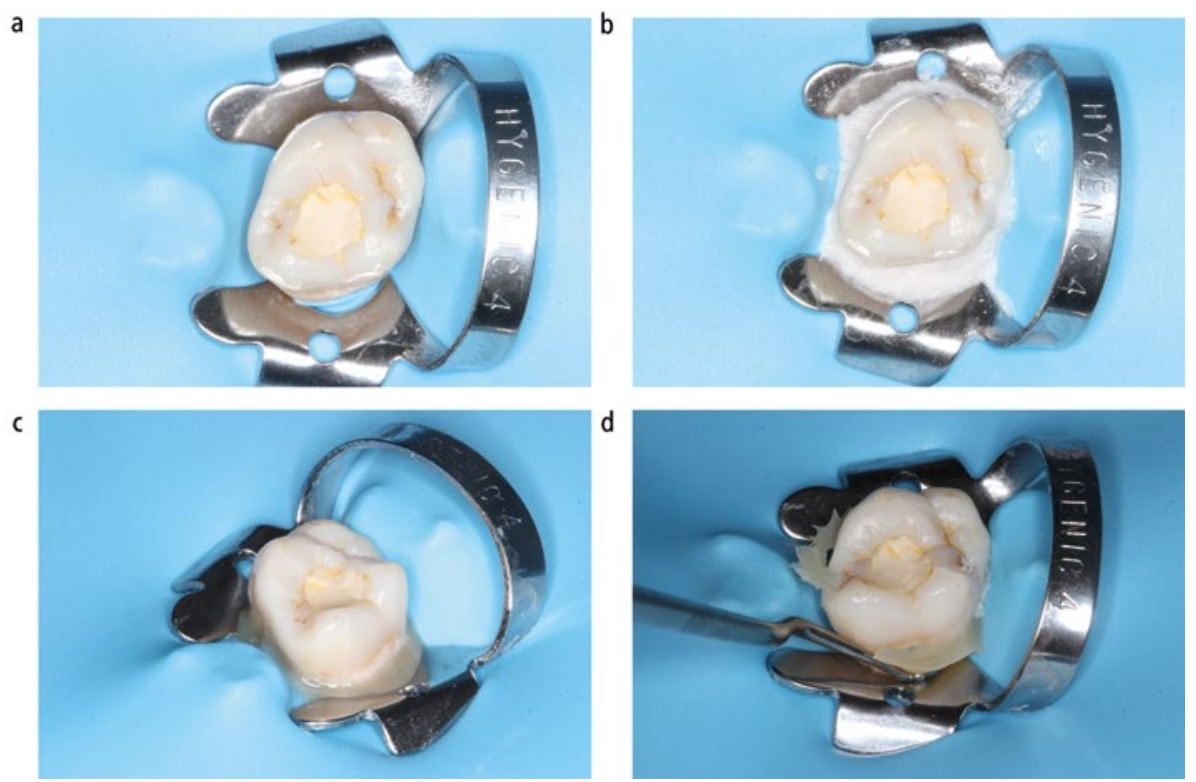

Fig. 4 a) Tooth isolated with rubber dam. b) Additional isolation achieved with caulking agent applied between the rubber dam and tooth. c) Tooth isolated with additional flowable composite placed at the margin between the clamp and tooth. The cured composite may also aid in stabilising the rubber dam clamp. d) The flowable composite can be simply removed by placing an instrument beneath it and levering it off
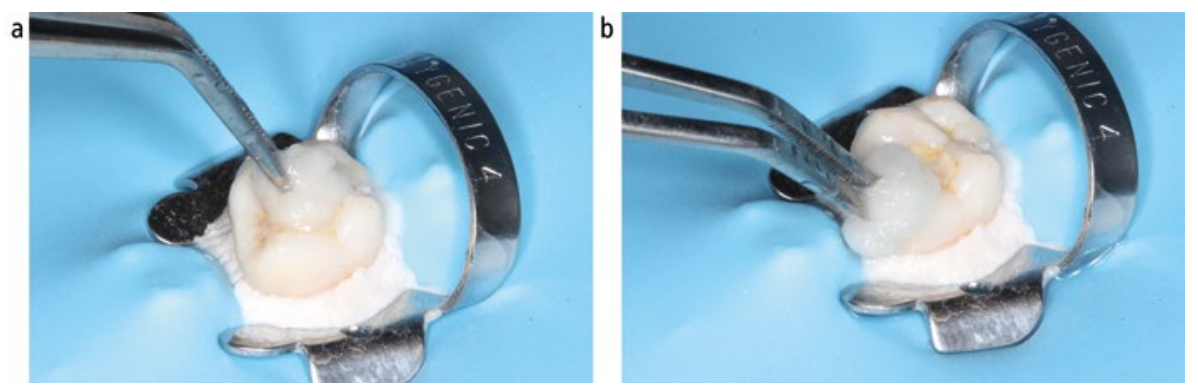

Fig. 5 Tooth scrubbed with cotton wool pledget soaked in sodium hypochlorite

- Rubber dam isolation is imperative; this should cover both the nose and oral cavity to reduce contamination with oral saliva and blood. Rubber dam isolation has been shown to reduce microorganisms by up to $98 \%$ when preparing teeth for direct restorations $\mathrm{s}^{29,30}$

- Ideally, load the dam for a single tooth with the appropriate clamp before placing into the mouth to reduce potential for aerosol. Utilise sealant materials if necessary to optimise the seal between the tooth and rubber dam. This can be achieved with a caulking agent, rubber dam liquid sealants or flowable composite, which may be more readily available (Fig. 4)

- Following isolation with rubber dam, the isolated tooth/teeth should be scrubbed with sodium hypochlorite, hydrogen peroxide or PVP-I utilising a cotton wool pledget and tweezers for one minute. Classic endodontic microbiology studies have sterilised the crown of a tooth before access to prevent cross infection of the pulpal microflora; this principle can be applied to the current situation. ${ }^{31}$ In comparison, prophying the tooth is likely to result in aerosol (Fig. 5)

- Once the tooth is disinfected, proceed to cleanse the area of the rubber dam local to the tooth in the same manner

- To begin your access cavity, utilise a fast handpiece with reduced water or without water and high-volume suction to reduce aerosol production. This is a deviation from normal practice in abnormal circumstances. Tungsten carbide burs cut more efficiently than diamond burs and hence are less likely to result in 

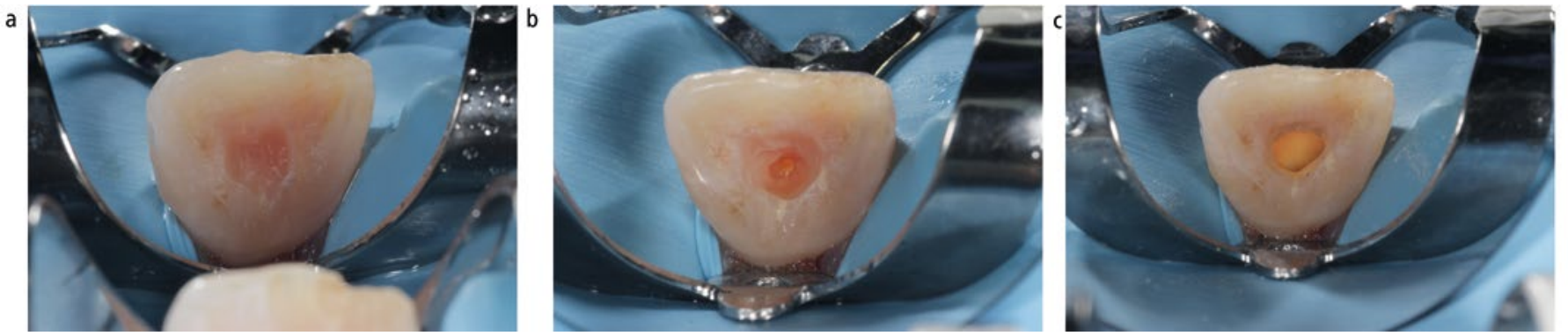

Fig. 6 a) Dentine breached with high-speed handpiece. b) Pulp breached utilising rose head bur in slow handpiece. c) Completion of access with slow handpiece

haphazard debris. There is only a need to remove the enamel within the ideal access cavity - once the dentine is breached switch to using a slow handpiece. Caries can be hand-excavated if necessary (Fig. 6)

- Ensure that high-volume suction is used as close to the tooth and the handpiece head as possible during drilling to reduce surface contamination with aerosol. The use of high-volume suction has been shown to reduce aerosol surface contamination between $90-93 \% \%^{32,33}$

- Refrain from the use of 3-in-1 syringe as much as possible; debris collected within the pulp system can be removed through highvolume suction. A fine surgical aspirator tip can be attached to aid application into the cavity

- Avoid the use of ultrasonic scalers, which have high aerosol production ${ }^{20}$

- Make use of Gates Glidden burs and/ or Goose Neck burs to improve access depending on the depth of the dentine overlying the pulp roof and the proximity of the canal orifices

- Once the pulp has been accessed, the inflamed tissue in the pulp chamber can be removed - essentially a pulpotomy. Next, 5\% sodium hypochlorite can be utilised to fully dissolve any organic tissue to the best possible level and minimise constant need for irrigation and re-irrigation ${ }^{34,35}$

- An alternative to the pulpotomy could be a 'pulpectomy' if time and equipment allow. The root canal system can be mechanically instrumented to length, with the aim of delivering irrigant and intracanal medicament into the root canal system to eradicate bacteria ${ }^{36}$

- Pulpotomies can be considered for irreversible pulpitis as the radicular pulp
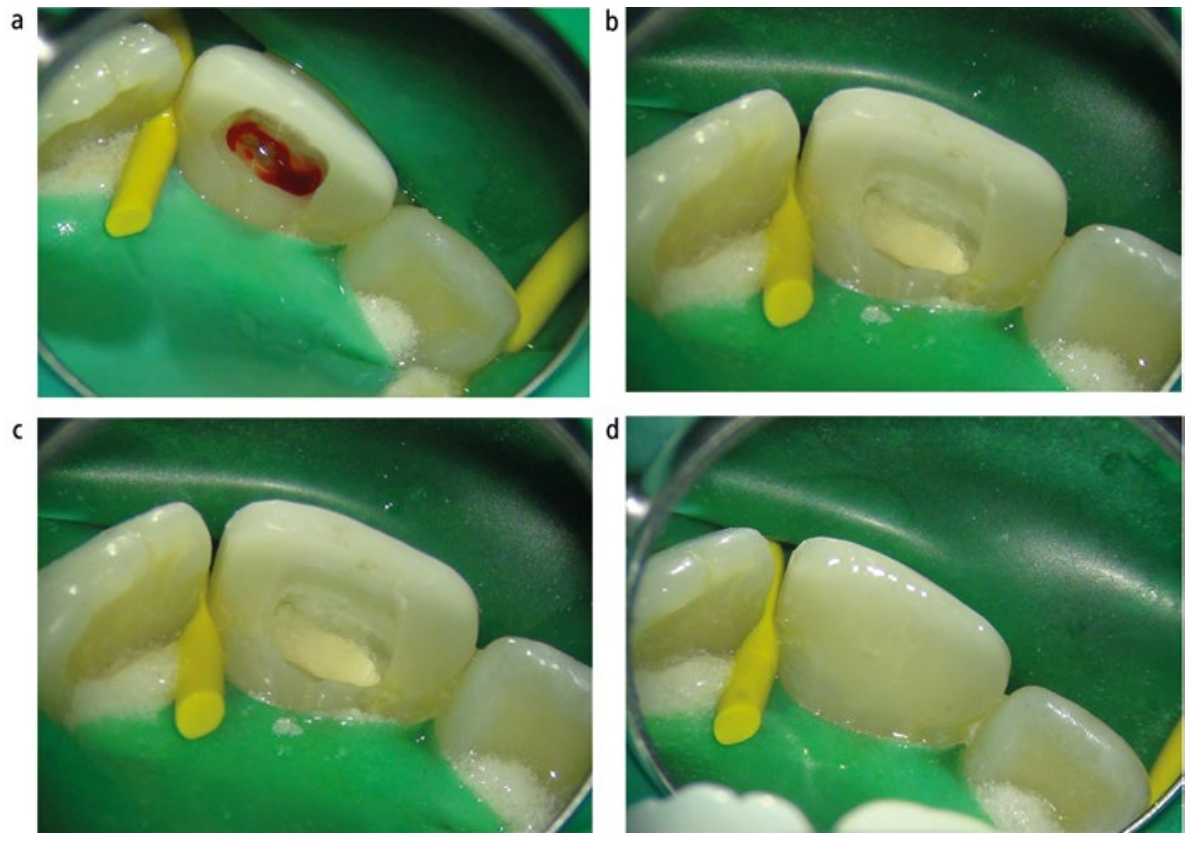

Fig. 7 a) Inflamed pulp tissue in a maxillary central incisor. b) Cessation of bleeding and healthy pulp visible. c) Placement of bioceramic material over the pulp tissue - Biodentine (Septodent, Saint Maur des Fosses, France) was used in this instance. d) Cavity restored with composite resin

is usually vital. Conversely, in necrotic cases, there is a well-established infection and the intention is to eliminate the microorganisms within the root canal system $^{37}$

- Wherever possible, single-visit root canal treatment should be provided. This will reduce the need for a further appointment as well as further risk of $\mathrm{AGPs}^{38}$

- If the clinician is unable to complete the root canal treatment, which is likely to be the majority of acute cases, dress the pulp system with an appropriate medicament. Consider non-setting calcium hydroxide for necrotic pulps and steroid/antibiotic pastes for cases of irreversible pulpitis

- Ideally, the tooth should be restored definitively with a direct restoration under rubber dam isolation if endodontics has been completed. This may also provide the foundations for an extra-coronal restoration at a later date and further reduce the burden of subsequent AGPs.

\section{Post-operative}

Depending on the patient's presenting symptoms, analgesics could be prescribed. The patient will need to be informed that the treatment provided is not definitive and will require follow-up. Appropriate advice should be given regarding delays in treatment in the current circumstances. This could impact outcomes of endodontic treatment - the patient may encounter further flare-ups of pain, a higher chance of tooth fracture and loss of temporary restoration. ${ }^{39}$ 


\section{Dental trauma management}

Avulsion and luxation injuries will require repositioning and treatment commonly in the form of a splint. Complicated crown fractures with pulpal involvement will also require treatment.

If teeth require splinting, then consideration can be given to the use of a composite resin using self-etching adhesive or a resin-modified glass ionomer cement. This would minimise the use of the 3-in-1 syringe and in turn reduce aerosol production.

Splint removal may be facilitated by sectioning the wire splint using pliers or wire cutters between the restorations. The composite buttons will remain on the tooth but can be removed at a later date; the remaining sections of wire or rough sections of composite can be lightly polished with hand abrasive strips and discs or abrasive discs in a slow handpiece to remove any sharp edges. ${ }^{40}$ This again would negate the use for the high-speed handpiece and in turn would reduce aerosol generation. In other circumstances, the clinician may decide that the flexible splint can remain in situ for the time being.

Managing complicated crown fractures provides a further challenge. Rubber dam should be applied and the tooth cleansed in the same manner as described above for endodontic treatment. Once isolated, the exposed pulp can be assessed for either a direct pulp cap (to minimise AGPs) or a partial pulpotomy. The provision of a partial pulpotomy should limit the amount of aerosol generation, as the length of instrumentation is unlikely to be prolonged in reaching healthy pulp tissue (Fig. 7a). Once healthy pulp tissue is exposed, haemostasis can be aided by a sterile cotton wool pledget soaked in sodium hypochlorite or saline (Fig. 7b). If haemorrhage does not cease, further tissue may need to be resected until haemostasis is achieved. The exposed healthy tissue can then be covered with non-setting calcium hydroxide or bioceramic materials such as mineral trioxide aggregate or calcium silicate-based materials (Fig. 7c). This can then be overlaid with glass ionomer cement or a composite restoration using a selfetching adhesive without the creation of further aerosol (Fig. 7d).

More in-depth guidance has been released regarding trauma management pertinent to COVID-19, which recommends that if a patient is seen, gold standard treatment should be provided, even if AGPs are likely to be involved. ${ }^{40}$ Where possible, this should be adhered to, otherwise it may lead to poorer outcomes and re-attendance.

\section{Discussion}

With dentistry being reduced to the bare essentials across the UK and reduction in patients being seen, the initial strategy of the AAA principle is helpful for a vast number of patients, with the first contact being through telephone triage.

The implications of this approach to emergency dental care are far-reaching and significant to patients and NHS dental services. Telephone triage by a professional through careful questioning on signs and symptoms, despite best efforts, may not provide a clear clinical picture of the problem without physical or radiographic investigation. The concern is that antibiotics and analgesics may be prescribed to individuals that may not require them or indeed are provided for conditions for which they may not be effective. A further consideration is what seems to be a wholesale change in the approach to antibiotic prescription at this clearly difficult time for the NHS. The National Institute for Health and Care Excellence has published guidelines aimed at managing over-prescription of antibiotics, in order to prevent antibiotic resistance and its implications. ${ }^{41}$ Such practices may result in unfortunate, unintended consequences for the patient in their future healthcare management.

The issue and concern for patients in which this is ineffective is that the result is repeat symptoms and morbidity. Antibiotics, without follow-on care such as the provision of endodontics or extraction (if indicated), can result in antibiotic resistance, potentially making any future management more challenging. As such, we are likely to be managing the legacy of this unfortunate period for many years to come. Indeed, patients who undertake the AAA route for management are more likely to be keen on accessing endodontic treatment as opposed to extraction, in the hope of maintaining the tooth.

The funding issues surrounding NHS endodontics have long been cited. ${ }^{42}$ As a result of the widespread antibiotic provision and the likely antimicrobial resistance that follows, we may see a huge rise in the need for endodontic services, and consequently, an increase in strain on the already sporadic access to secondary care endodontics for teeth referred for de novo or root canal re-treatment. ${ }^{43}$

\section{Acknowledgements}

The authors would like to thank John Rhodes, Specialist Endodontist, Poole, UK for the images used in Figure 7.
Conflict of interest

All authors declare they have no conflict of interest.

\section{References}

1. Peng $X, X u X, L i Y$, Cheng L, Zhou X, Ren B. Transmission routes of 2019-nCoV and controls in dental practice. Int J Oral Sci 2020; 12: 1-6.

2. European Centre for Disease Prevention and Control. COVID-19 pandemic. 2020. Available at https://www. ecdc.europa.eu/en/covid-19-pandemic (accessed May 2020).

3. Lu C W, Liu X F, Jia Z F. 2019-nCoV transmission through the ocular surface must not be ignored. Lancet 2020; DOI: 10.1016/S0140-6736(20)30313-5.

4. Cascella M, Rajnik M, Cuomo A, Dulebohn S C, Di Napoli R. Features, evaluation and treatment coronavirus (COVID-19). In StatPearls [Internet]. Treasure Island (Florida): StatPearls Publishing, 2020.

5. UK Government. Coronavirus (COVID-19) cases in the UK. Available at https://coronavirus.data.gov.uk/ (accessed May 2020)

6. World Health Organisation. Infection prevention and control of epidemic- and pandemic-prone acute respiratory infections in health care. 2014. Available at https://apps.who.int/iris/bitstream/ handle/10665/112656/9789241507134_eng. pdf? sequence $=1$ (accessed June 2020).

7. Harrel S K, Molinari J. Aerosols and splatter in dentistry: a brief review of the literature and infection control implications. J Am Dent Assoc 2004; 135: 429-437.

8. Office for National Statistics. Which occupations have the highest potential exposure to the coronavirus (COVID-19)? 2020. Available at https://www.ons.gov. uk/employmentandlabourmarket/peopleinwork/ employmentandemployeetypes/articles/which occupationshavethehighestpotentialexposuretothe coronaviruscovid19/2020-05-11 (accessed June 2020).

9. Public Health England. Guidance: COVID-19 personal protective equipment (PPE). 2020. Available at https:// www.gov.uk/government/publications/wuhan-novelcoronavirus-infection-prevention-and-control/covid19-personal-protective-equipment-ppe (accessed June 2020).

10. NHS Scotland. Assessing the evidence base for medical procedures which create a higher risk of respiratory infection transmission from patient to healthcare worker. 2020. Available at https://hpspubsrepo. blob.core.windows.net/hps-website/nss/3055/ documents/1_agp-sbar.pdf (accessed June 2020).

11. Van Doremalen N, Bushmaker T, Morris D H et al. Aerosol and surface stability of SARS-CoV-2 as compared with SARS-CoV-1. N Engl J Med. 2020; 382: 1564-1567.

12. Chief Dental Officer. Issue 3: Preparedness letter for primary dental care - 25 March 2020. 2020. Available at https://www.england.nhs.uk/coronavirus/wp-content/ uploads/sites/52/2020/03/issue-3-preparednessletter-for-primary-dental-care-25-march-2020.pdf (accessed April 2020).

13. Chief Dental Officer. Issue 4: Preparedness letter for primary dental care - 15 April 2020. 2020. Available at https://www.england.nhs.uk/coronavirus/wp-content/ uploads/sites/52/2020/03/C0282-covid-19-dentalpreparedness-letter-15-april-2020.pdf (accessed April 2020).

14. NHS England. COVID--19 guidance and standard operating procedure. 2020. Available online at https:// www.england.nhs.uk/coronavirus/publication/ covid-19-guidance-and-standard-operatingprocedure-urgent-dental-care-systems-in-the-contextof-coronavirus/ (accessed April 2020).

15. Chief Dental Officer. Resumption of Dental Services in England. 2020. Available at https://www. england.nhs.uk/coronavirus/wp-content/uploads/ sites/52/2020/03/Urgent-dental-care-letter-28-May. pdf (accessed June 2020)

16. Office of Chief Dental Officer England and NHS England. Standard operating procedure: Transition to recovery. 2020. Available at https://www.england.nhs.uk/coronavirus/ wp-content/uploads/sites/52/2020/06/ 
C0575-dental-transition-to-recovery-SOP-4June.pdf (accessed June 2020)

17. Scottish Dental Clinical Effectiveness Programme. Management of Acute Dental Problems During COVID19 Pandemic. 2020. Available at http://www.sdcep. org.uk/wp-content/uploads/2020/03/SDCEP-MADPCOVID-19-guide-300320.pdf (accessed April 2020).

18. British Endodontic Society. Diagnosis and Management of Endodontic Emergencies, a British Endodontic Society Position Paper for Primary Dental Care and other healthcare providers during the COVID-19 pandemic. 2020. Available at https://britishendodonticsociety. org.uk/wp-content/uploads/2020/04/BES-EmergencyProtocol-v3-April-23-1.pdf (accessed May 2020).

19. Public Health England. COVID-19: personal protective equipment use for aerosol generating procedures. 2020. Available online at https://www.gov.uk/ government/publications/covid-19-personalprotective-equipment-use-for-aerosol-generatingprocedures (accessed May 2020).

20. Bennett A M, Fulford M R, Walker J T, Bradshaw D J, Martin M V, Marsh P D. Microbial aerosols in general dental practice. Br Dent J 2000; 189: 664-667.

21. Public Health England. COVID-19: infection prevention and control guidance. 2020. Available at https:// assets.publishing.service.gov.uk/government/uploads/ system/uploads/attachment data/file/893320/COVID19_Infection_prevention_and_control_guidance_ complete.pdf (accessed May 2020).

22. Faculty of General Dental Practice (UK) and College of General Dentistry. Implications of COVID-19 for the safe management of general dental practice: A practical guide. 2020. Available at https://www.fgdp.org.uk/ sites/fgdp.org.uk/files/editors/Implications\%20of\%20 COVID-19\%20for $\% 20$ the $\% 20$ safe $\% 20$ management $\% 20$ of $\% 20$ general $\% 20$ dental $\% 20$ practice $\%$ C $2 \%$ A0- $\% 20$ \%20practical\%20guide.pdf (accessed June 2020).

23. Rees J. Asepsis in implant dentistry. Prim Dent J 2012; 1: 47-49.
24. Kampf G, Todt D, Pfaender S, Steinmann E. Persistence of coronaviruses on inanimate surfaces and its inactivation with biocidal agents.J Hosp Infect 2020; 104: 246-251.

25. Eggers M, Koburger-Janssen T, Eickmann M, Zorn J. In vitro bactericidal and virucidal efficacy of Povidonelodine gargle/mouthwash against respiratory and oral tract pathogens. Infect Dis Ther 2018; 7: 249-259.

26. Kawana R, Kitamura T, Nakagomi 0 et al. Inactivation of human viruses by povidone-iodine in comparison with other antiseptics. Dermatology 1997; DOI: 10.1159/000246027.

27. Farzan A, Firoozi P. Common Mouthwashes for PreProcedural Rinsing in Dental Practice: Which One Is Appropriate For Eliminating Coronaviruses? A Mini Literature Review. J Regener Reconstr Restor 2020; DOI: 10.22037/rrr.v5i1.29543.

28. Kirk-Bayley J, Challacombe S, Sunkaraneni S, Combes J. The Use of Povidone lodine Nasal Spray and Mouthwash During the Current COVID-19 Pandemic May Protect Healthcare Workers and Reduce Cross Infection. 2020. Available online at https://papers.ssrn.com/sol3/papers. cfm?abstract_id=3563092 (accessed March 2020).

29. Cochran M A, Miller C H, Sheldrake M A. The efficacy of the rubber dam as a barrier to the spread of microorganisms during dental treatment. J Am Dent Assoc 1989; 119: 141-144.

30. Samaranayake L P, Reid J, Evans D. The efficacy of rubber dam isolation in reducing atmospheric bacterial contamination. ASDC J Dent Child 1989; 56: 442-444.

31. Byström $A$, Sundqvist $G$. Bacteriologic evaluation of the efficacy of mechanical root canal instrumentation in endodontic therapy. Scand J Dent Res 1981; 89: 321-328.

32. Harrel S K, Barnes J B, Rivera-Hidalgo F. Reduction of aerosols produced by ultrasonic sealers. J Periodonto 1996; 67: 28-32.

33. Jacks M E. A laboratory comparison of evacuation devices on aerosol reduction. J Dent Hyg 2002; 76: 202-206.
34. Hand R E, Smith M L, Harrison J W. Analysis of the effect of dilution on the necrotic tissue dissolution property of sodium hypochlorite. J Endod 1978; 4: 60-64.

35. Stojicic S, Zivkovic S, Qian W, Zhang H, Haapasalo M. Tissue dissolution by sodium hypochlorite: effect of concentration, temperature, agitation, and surfactant. J Endod 2010: 36: 1558-1562.

36. Hülsmann M, Peters O A, Dummer P M. Mechanical preparation of root canals: shaping goals, techniques and means. Endod Topics 2005; DOI: 10.1111/j.1601-1546.2005.00152.x.

37. Siqueira Jr J F, Rôças I N. Clinical implications and microbiology of bacterial persistence after treatment procedures. J Endod 2008; 34: 1291-1301.

38. British Endodontic Society. BES COVID-19: Return to Work SOP. 2020. Available at https://britishendodonticsociety. org.uk/wp-content/uploads/2020/06/BES_SOP080620-v1.pdf (accessed June 2020).

39. Ng Y L, Mann V, Rahbaran S, Lewsey J, Gulabivala K. Outcome of primary root canal treatment: systematic review of the literature - Part 2. Influence of clinical factors. Int Endod J 2008; 41: 6-31.

40. Dental Trauma UK and British Society of Paediatric Dentistry. Permanent Dentition Acute Management of Traumatic Injuries and Follow-up Care during the COVID-19 Pandemic. 2020. Available at https://www. dentaltrauma.co.uk/File. ashx?id=15336 (accessed June 2020)

41. National Institute for Health and Care Excellence. Antimicrobial stewardship: systems and processes for effective antimicrobial medicine use. 2015. Available at https://www.nice.org.uk/guidance/NG15/chapter/1Recommendations\#recommendations-for-prescribers (accessed April 2020).

42. Alani A, Bishop K. Contemporary issues in the provision of restorative dentistry. Br Dent J 2012; 213: 163-170.

43. Ng Y-L, Mann V, Gulabivala K. Outcome of secondary root canal treatment: a systematic review of the literature. Int Endod J 2008; 41: 1026-1046.

\section{Correction to: The changing face of dental practice: emerging models of team care in Australia}

The original article can be found online at https://doi.org/10.1038/s41415-020-1529-3.

\section{Author's correction note:}

Research article Br Dent J 2020; 228: 767-772.

When this article was published there were errors in Figures 1 and 2.

The correct version of Figures 1 and 2 are presented here:

The original article has been corrected.

\section{Fig. 1 Child models of care}

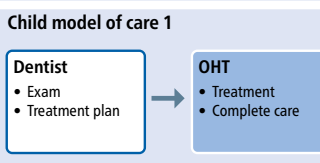

Child model of care 2

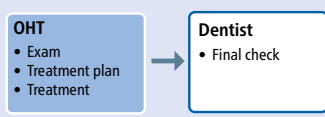
Child model of care 3

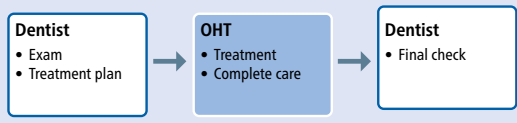

Child model of care 4

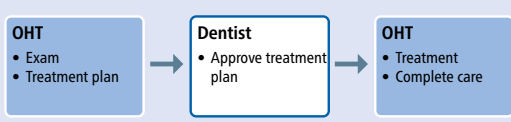

Child model of care 5 OHT - Exam - Exam
- Treatment plan
- Treatment - Complete care ete car

\section{Fig. 2 Adult models of care}

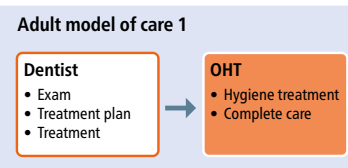

Adult model of care 2

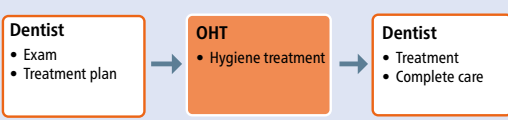

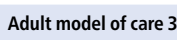

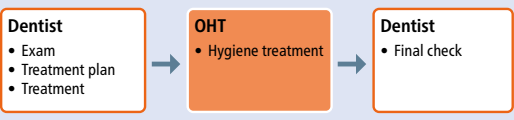

Adult model of care 4
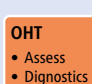
- Assess - Hygiene treatm

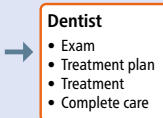

